The Journal of

Thoracic and Cardiovascular

Surgery

Vol 131, No. 1, January 2006

\title{
An "actual" problem: Another issue of apples and oranges
}

Endre Bodnar, MD, and Eugene $\mathrm{H}$. Blackstone, $\mathrm{MD}^{\mathrm{b}}$

"The road to hell is paved with good intentions"

(Proverb)

Editor in Chief, The Journal of Heart Valve Disease $^{\mathrm{a}}$; Director of Clinical Research, Department of Thoracic and Cardiovascular Surgery, and Department of Quantitative Health Sciences, ${ }^{\mathrm{b}}$ The Cleveland Clinic Foundation, Cleveland, Ohio.

Reprinted with permission from The Journal of Heart Valve Disease 2005;14:706-8.

Received for publication Nov 1, 2005; accepted for publication Nov 22, 2005.

Address for reprints: Endre Bodnar, MD, Crispin House, 12/A South Approach, Moor Park, Northwood, HA6 2ET, United Kingdom (E-mail: bodnarendre@aol.com).

J Thorac Cardiovasc Surg 2006;131:1-3

$0022-5223 / \$ 32.00$

Copyright $\odot 2006$ by ICR Publisher

doi:10.1016/j.jtcvs.2005.11.013
$\mathrm{W}$ hen deliberating on replacement heart valves, two important questions spring to mind. One is, "How does the replacement valve perform in terms of time-related probability of occurrence of failure and other complications attributable to the device itself?" Answering this question is the basis for making valid comparisons among different types and models of prosthesis. The second is, "How likely is it that a patient with a replacement device will survive to experience a given device-related complication?" Because the make-up (patient mix or profile) of various groups of patients receiving heart valves differs with respect to risk factors for mortality, the number of patients living to experience a given complication will differ and will not be comparable, even though the attributes of the device remain the same.

Clearly these two questions differ, as do their answers; one addresses intrinsic properties of the device; the other places these properties into the context of specific patients or groups of patients. One is apple, the other is orange, and they are not to be confused.

Actuarial analysis, ${ }^{1}$ whether by the life table method, ${ }^{2}$ Kaplan-Meier product limit calculation, ${ }^{3}$ or a number of other variants, was originally introduced to assess survival probability in a population where not everybody is dead at the time of inquiry. ${ }^{4}$ The method offered itself as eminently suitable when the time-related assessment of non-fatal events became a necessity. ${ }^{5}$ In this case, however, there is a caveat. Due to its underlying statistical and mathematical basis, actuarial assessment of a non-fatal event assumes that the entire patient population will live forever. At first blush, this assumption is preposterousnobody lives forever! However, it may not seem so preposterous if stated in another, completely equivalent way: "We assume that patients who die before a non-fatal event occurs were just as likely, while they were alive, as anybody else to have experienced that event, even though they didn't." Now, that sounds more sensible, doesn't it?

Why should we be interested in this? One of the most useful strategies for laboratory investigation of a complex phenomenon is to hold all things constant and vary one thing at a time. Thus, if there are many reactions competing for a single substrate and we want to understand the characteristics of one of the reactions, we design the experiment to stop all other reactions except the one of interest. Patients are mortal, and their life and well-being are threatened continuously by a broad number of conditions, just as in an uncontrolled laboratory study. A replacement valve is only one of these conditions thrown into an environment of multiple competing risks. Despite this, can we isolate the properties of the device, holding all these competing risks constant, as in a controlled environ- 
ment? This question can be crucial! Actuarial analysis is exactly the methodology we need, because it isolates one event at a time from all others, such as the attributes of a prosthesis.

On the other hand, considering an individual patient, another question arises: "Which is more likely to occur first, failure of the implanted valve or death?" Indeed, this question also can be crucial! About 15 years ago, whether or not elective reoperation was indicated to prevent strut fracture of the BjörkShiley valve was just such a question. In 1992, Blackstone and Kirklin $^{6}$ worked out the multivariable equations to advise clinicians on which individual patient should and which should not be offered elective removal of the valve based on which competing event, death or strut fracture, was likely to occur first (this was the first article published in the first issue of The Journal of Heart Valve Disease).

Two years later, Grunkemeier et $\mathrm{al}^{7}$ asked a simpler question: "What is the likelihood that a given patient will or will not suffer a valve-related complication or ultimate failure of the implanted valve?" They used cumulative incidence to answer the question, considering the probability of death of the patient and that of the valve-related event as competing risks. Calculation of cumulative incidence is a well-established statistical mathematical method, and its application in the given context was perfectly correct.* Other authors went, however, subsequently two steps further:

1. They displayed graphically the complement of cumulative incidence, which normally rises from zero to a certain positive value, so that the new curve declined from $100 \%$ to a certain value.

2. They named this new curve the actual freedom from a valve-related event; particularly, actual freedom from structural degeneration of a bioprosthetic valve. (Note that Grunkemeier et al used the term actual risk rather than actual freedom.)

Actuarial and actual curves were superimposed on the same vertical axis, even though one represented a probability and the other an upside-down cumulative incidence. The apples and oranges comparison led to a considerable amount of confusion. Grunkemeier et al clearly stated that actual risk is not a statistical term, let alone actual freedom from an event, and it should be used only (a) to advise an individual patient or (b) to make some socioeconomic assumptions regarding use of a given device in a certain patient population. Regrettably, it was not stated firmly that it must not be used to define and compare intrinsic valve performances.

\footnotetext{
*Cumulative incidence dates back to Daniel Bernoulli in the 18th century, who just a few years before Jenner inoculated 8-year-old James Phipps with cow pox, contemplated how the population would change if the competing risk of death from smallpox were eradicated. ${ }^{8}$ Since then, it has been reinvented multiple times and given multiple names. Perhaps its first use in heart valve disease was by Bodnar and colleagues in $1979,{ }^{9}$ who used the term "multiple decrement" analysis, as introduced by demographers.
}

This led to the Cleveland Clinic reporting actuarial and actual freedom from valve-related events with the CarpentierEdwards Perimount valve in $1998 .{ }^{10}$ With this, the genie was out of the bottle.

As per definition, so-called "actual freedom" (which is not a statistical term, vide supra) is always higher than actuarial freedom, because the former is influenced by the non-valve-related mortality of the patient population (that is, for some patients, death comes before valve explant). Taking the simplest example, the older the patient, the larger the difference between "actual" and actuarial freedom. As a consequence, so-called "actual" freedom has been appearing with increasing frequency in publications reporting valve performance and has been used by some manufacturers to promote their product. The state of affairs has become so distorted that there are members (not statisticians) of the medical profession who currently insist that both actuarial and actual freedoms be given in every publication reporting long-term performance of replacement valves. Medical science is suffering a catastrophic blow, and we must act quickly and decisively to avoid further damage.

\section{What Should Be Done?}

1. We should stop using the term "actual freedom." Synonyms of "actual" are current, eventual, and real, and the implication of its use is that it is more real than the actuarial estimate. There is no reason to abandon the expression cumulative incidence if that is what the authors intend to report, but it must be used in the proper context.

2. Cumulative incidence (since 1994 called "actual" freedom in heart valve publications) must not be used to define or compare valve performance. This should be done using actuarial methods.

3. All so-called "actual freedom" results published in the medical literature or in commercial files should be completely disregarded as invalid descriptions of valve performance. Only actuarial results should be used to describe device performance. Any statement of device performance based on "actual freedom" from valve-related events should be considered scientifically invalid and potentially misleading.

In conclusion, the Journal will no longer publish "actual freedom" results in articles reporting long-term performance of replacement valves. We will ask our authors to provide cumulative incidence if relevant, but not as a measure of durability or quality of a replacement device, for which actuarial results are required. This approach must become consistent in scientific publications and in advertising material. ${ }^{8,9}$ 


\section{References}

1. Blackstone EH. Actuarial and Kaplan-Meier survival analysis: there is a difference. J Thorac Cardiovasc Surg. 1999;118:973-5.

2. Cutler SJ, Ederer F. Maximum utilization of the life table method in analyzing survival. J Chronic Dis. 1958;8:699-712.

3. Kaplan EL, Meier P. Nonparametric estimation from incomplete observations. J Am Stat Assoc. 1958;53:457-81.

4. Berkson J, Gage RP. Calculation of survival rates for cancer. Mayo Clin Proc. 1950;25:270.

5. Blackstone EH, Kirklin JW. Death and other time-related events after valve replacement. Circulation. 1985;72:753-67.

6. Blackstone EH, Kirklin JW. Recommendations for prophylactic removal of heart valve prostheses. J Heart Valve Dis. 1992;1:3-14.
7. Grunkemeier GL, Jamieson WR, Miller DC, Starr A. Actuarial versus actual risk of porcine structural valve deterioration. $J$ Thorac Cardiovasc Surg. 1994;108:709-19.

8. Bernoulli D. Essai d'une nouvelle analyse de la mortalité cause par la petite Vérole, et des avantages de l'Inoculation pour la prévenir. Mém de l'Académie Royale de Science, 1760, 1-45.

9. Bodnar E, Haberman S, Wain WH. Comparative method for actuarial analysis of cardiac valve replacements. Br Heart J. 1979;42: 541-52.

10. Banbury MK, Cosgrove DM 3rd, Lytle BW, Smedira NG, Sabik JF, Saunders CR. Long-term results of the Carpentier-Edwards pericardial aortic valve: a 12-year follow-up. Ann Thorac Surg. 1998;66(6 Suppl): S73-6. 